\title{
Canadian Normative Data for Minimal Assessment of Cognitive Function in Multiple Sclerosis - CORRIGENDUM
}

\author{
Lisa A.S. Walker, David Marino, Jason A. Berard, Anthony Feinstein, \\ Sarah A. Morrow, Denis Cousineau
}

Keywords: Multiple sclerosis, cognition, neuropsychological tests, psychometrics, Canada, corrigendum

doi: https://doi.org/10.1017/cjn.2017.199, Published by Cambridge University Press, 7 July 2017

The above article ${ }^{1}$ published with multiple errors.

On page 549, second paragraph after the section heading "Regression-Based Norms", the sentence should read "...CVLT-II Total Recall (education); CVLT-II List B Free Recall (education); CVLT-II Short Delay Free Recall (age); CVLT-II Long Delay Free Recall (age, education); CVLT-II Long Delay Cued Recall (age); CVLT-II, Total Intrusions (age, education); CVLT-II, Total Repetitions (age); BVMT-R Total Recall (age)..."

On page 551, Table 3, in the row labelled "SDMT" and in the column labelled "Regression-based normative formulae", the entry should read "70.62 + (sex x 2.28) - (age x 0.36) + (educ x 0.095)".
On page 552, right-hand column, third line from the bottom, the sentence should read "Performance on the nonverbal memory test (BVMT-R) was similar to that on the verbal task, with age being most contributory".

The authors apologize for these errors.

\section{REFERENCE}

1. Walker LAS, Marino D, Berard JA, Feinstein A, Morrow SA, Cousineau D. Canadian normative data for minimal assessment of cognitive function in multiple sclerosis. Can J Neurol Sci. 2017;44(5):547-5. 